\title{
A Flexible IT Infrastructure for Integrated Urban Planning Wout Hofman ${ }^{1}$, Walter Lohman ${ }^{2}$ and Ab Schelling ${ }^{3}$
}

\author{
TNO, Delft, ${ }^{1}$ wout.hofman@tno.nl, ${ }^{2}$ walter.lohman@tno.nl, ${ }^{3}$ ab.schelling@tno.nl \\ ${ }^{3}$ Technical University Delft
}

Received 06 August 2010; received in revised form 14 November 2010; accepted 22 December 2010

\begin{abstract}
This paper presents an IT infrastructure based on an event driven architecture with the objective to decrease the turnaround time for urban planning. Most urban planning takes a long time, not only to get all stakeholders involved, but also to assess various scenarios on several aspects according to (inter)national laws and regulations. By supporting urban planning with IT, the turnaround time is expected to decrease dramatically and supports the possibility to explore more scenarios without an extra burden on duration and personnel. The IT infrastructure developed integrates various data resources and calculation models for analyzing different scenarios. An event service bus and a data store for sharing data between computational models is the basis of the infrastructure. Various computational models are able to read and write to the data store and publish and subscribe to events. Based on a case study, the paper illustrates that indeed decision making is improved leading to better and possibly cheaper urban plans for all relevant stakeholders. The paper will also show that a closed environment, as currently available, is one of the major thresholds for acceptance by end-users. Endusers would like to apply their computational models, which can not yet be plugged into the infrastructure. Further extensions are foreseen in applying open standards and open link data for data collection and sharing. Thus, interoperability is a prerequisite for our IT infrastructure.
\end{abstract}

Keywords: Interoperability, Infrastructure, Flexibility, Event driven architecture, Urban planning 


\section{Introduction}

Cities can be viewed as dynamic socio-technical systems that interact with a continuously changing world [1]. Therein, the technical network and the actors and bodies of rules that are involved - the social network - together form an interconnected complex network (Figure 1). In the technical network material, energy, money and information are transformed and exchanged over suitable interfaces. The social network considers all stakeholders and their (in)formal relationships, e.g. contractors, architects, project developers, building societies and of course municipalities. These stakeholders all have vested interests in a city, e.g. because they own property or intend to exploit new property. Developments are ruled by various national and international regulations, e.g. EU regulations contain agreements regarding pollution that are guiding changes in cities and infrastructures. Cultural differences define how stakeholders participate in developments.

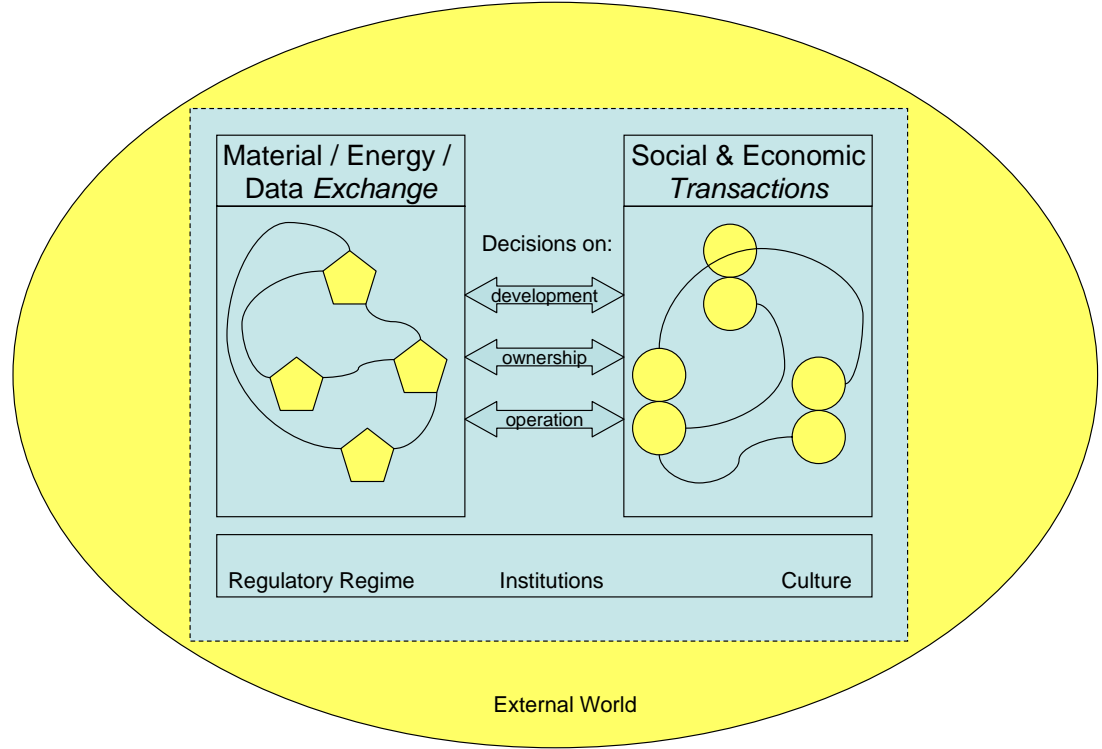

Figure 1: Dynamic socio-technical systems

Much like natural ecosystems, our cities can be seen to constantly evolve because individuals, organizations and governments decide on, for example, development, restructuring, demolition, transport infrastructure, energy grids, sustainable housing, shopping, (dis)investment, coordination \& regulation, respectively, in response to and in interaction with their respective environments. With time, cities evolve and complex structures emerge, which are characterized by diversity, multiple interactions both within and between layers, feedback loops and emergence - all characteristics that lead us to consider them as "complex". In such a complex system, control is distributed over the various actors - there is no single actor determining the configuration and behaviour of the system. With time, a system structure assembles and total system behaviour emerges [13].

Planning for cities today has additional environmental and social priorities in common with many topics that concern industrial ecology [1]. It implies for instance that urban development projects require an Environmental Impact Assessment (EIA, [1]) and Strategic Environmental Assessment (SEA, [1]) according to EU directives [11] and the United Nations/Economic Commission for Europe [24]. Environmental aspects that need to be included are direct and indirect effects of plans on (1) human beings, flora and fauna, (2) soil, water, air, climate and the landscape, (3) the interaction between the factors mentioned in the first and second indent, and (4) material assets and the cultural heritage. Composing an EIA or SEA may take a lot of time, e.g. three to six months per scenario due to its complexity and lack of IT support. Complexity is based on (a) all the aspects that need to be considered and (b) their interaction. Interaction considers data acquisition and calculation of impact of changes. Data required to analyse an environment and design a change, has to be gathered from various resources, e.g. a municipality has data representing a physical area and statistics aggregated data on its inhabitants with different levels of completeness and time scales. This data is provided in various formats according to (inter)national, de-facto and proprietary standards. Furthermore, the various models use different data in different formats. In the socio-technical system, each stakeholder again will have its proprietary formats. Thus a large interoperability issue is introduced. With respect to urban planning, the following models are considered:

- $\quad$ Traffic models that make predictions on traffic density, accessibility, etc. [24].

- $\quad$ Noise models that include various sources like traffic with its various modes (tram, railway, cars, [3], [3]) 
- Air quality models determining emission of air pollutants by for instance traffic and industry and effect on human health [13].

- External safety hazard model for the safety of population with respect to storage and transport of hazardous goods [25].

- $\quad$ Urban heat models to calculate the effect of climate changes [28].

- $\quad$ Sustainability models that calculate the impact of the previous models in the future [13].

The interaction between these models requires a complete new approach which makes it able to run as a grid [5]. A change calculated by a particular model may have impact on the calculation of yet another model. An Event Driven Architecture combined with a Service Oriented Architecture offers a solution, as each model subscribes to relevant events produced by other models and produce events itself [7]. This type of architecture is supported by publishsubscribe middleware that allows objects like organizations, models, or IT components to subscribe to certain events, produce events themselves, and obtain data by initiating services. In development, we followed a design-science paradigm by constructing a complete new artefact based on the integration of existing artefacts [16]. The models mentioned here were already existing artefacts. By constructing a new, innovative artefact that operates like a grid [5] and applying it in our problem domain, we achieved a better knowledge of our domain. As the focus of our research is to improve planning processes, we investigated other approaches in urban planning like computer-based simulations used for testing theories and interaction between land uses and related activities. In this respect, two classes of models can be distinguished: Land Use Transportation models incorporating macro and micro economics [14] as for instance implemented by UrbanSim [30] and Cellular Automata (CA), Agent-Based Models (ABM) and Micro-simulation involving models built around representing action and behaviour of individual agents located in space. There have been a substantial number of applications for the latter class of models, but few have been used to test urban policies in that transportation is handled rather crudely or even excluded and they largely ignore spatial economy such as house prices, wage rates, and transport costs [1].

We first describe urban planning, secondly, the architecture of our solution and finally a case. We end this contribution by giving conclusions and identifying issues for further research.

\section{Urban Planning and IT Support}

Urban planning processes, which are a specialization of spatial planning as they restrict to urban areas, are mostly structured in various activities. Each activity delivers a more detailed specification of an urban plan, e.g. from goal modelling to requirements analysis, design and actual realisation. Different stakeholders participate in each activity. Figure 2 shows a high level urban planning process of government organisations, participating stakeholders and deliverables. Probably, each government organization responsible for urban planning has a variation of this process with additional deliverables for each activity in the process. Design most often results in a framework defining the boundaries in which companies and citizens can realize constructions. Currently, a framework also comes with an urban plan visualizing a possible realization for communication purposes, e.g. a physical 3D model. Boundaries in a framework relate to physical dimensions of buildings (e.g. width and height), the use of buildings and areas, the locations in which constructions are allowed, traffic, noise, air pollution and other relevant parameters for a sustainable urban area. These boundaries are the starting point for private companies or individuals to develop their design as part of the realization of a plan. For realizing (parts of) an urban plan, a company or individual requires a permit. Currently, each deliverable in this urban planning process is a document containing relevant semantics, which make it impossible to visualize and construct an integrated view of all results in computer devices.

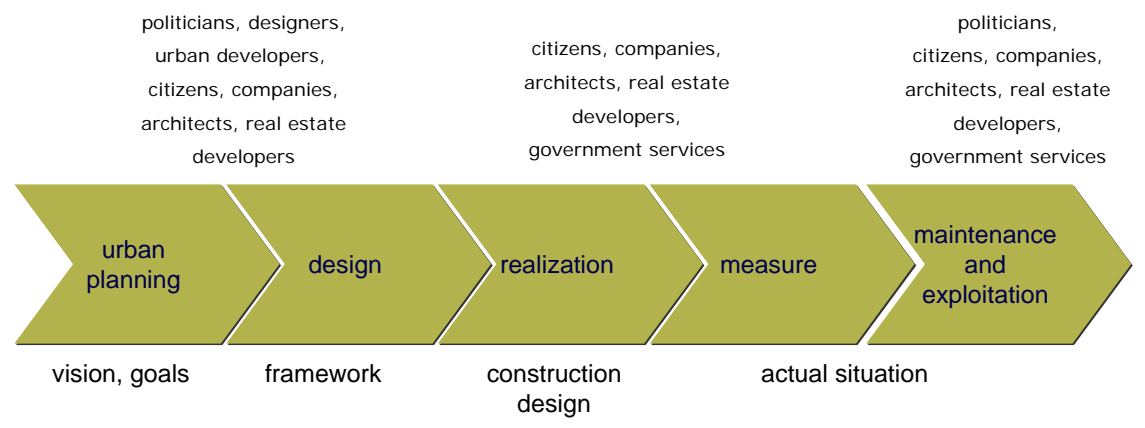

Figure 2: Activities, deliverables, and stakeholders in urban planning

IT support of this process focussed on administrative processes like permit processing and actual measurement of urban plans after their realization. Measurements were traditionally in two dimensions, but lately also 3D measurements are taken, e.g. using satellites [13]. It is foreseeable that IT with mobile devices can play a more 
important role in spatial planning and are already used in practice, e.g. Cadastre uses GPS devices to measure the actual situation.

Open standards to support all activities in urban planning are of growing importance for sharing information between all stakeholders for participation and decision making. Currently, CityGML is the open standard accepted by the Open Geo Consortium [28] and also supported by products of Google (e.g. Google Sketchup). CityGML consists of a class diagram of spatial objects like buildings and their artefacts like windows and the spatial representation of these objects as presented in GML (Geographical Markup Language). The standard supports levels of detail of urban areas relevant for rendering by geo-viewers. These levels of detail range from an overview of the area to a detailed 3D visualization of spatial objects and the interior of a building. CityGML is extendable for supporting different views like noise and air pollution by so-called application domain extensions. Integration between CityGML for visualization of a building and standards representing building construction (Building Information Model) is currently sought for calculating the actual impact of a new building on its environment, e.g. not only for safety but also for energy emission.

CityGML can be used to represent the actual situation or an urban plan, but does not support a framework and vision. A vision for instance contains rough sketches of potential changes in an area. These sketches have semantics which is mostly represented by a colour indicating the usage of a particular area (e.g. blue for water with a potential for leisure and red for build area for living, shopping, and leisure). The semantics and colour of a particular vision can differ per document. To support these differences, ontology is required that integrates a vision to a design represented in CityGML [22]. The Netherlands has specified a standard to represent two-dimensional content of spatial plans [20]. One could imagine that frameworks can be visualized by wire frames in for instance mobile devices with location awareness.

The environment presented in this paper supports any detailed design as can possibly also be represented in CityGML with a number of application domain extensions for each of the models. Level of detail 1 of CityGML, a block model, is sufficient to support the earlier mentioned models. Application domain extensions can be constructed to support the visualization of the results of models, but the models also need sufficient input like the number of inhabitants related to a type of building and the number of commuters. This type of information is not supported by CityGML or its extensions, but could be supported by ontology.

\section{Architecture}

The objective to create an IT infrastructure is to quickly assess the impact of an urban plan as required by international agreements and national laws and regulations. As we have indicated, such an urban plan can be represented by for instance CityGML. The core of the solution is based on the concept 'event'. There are many definitions of 'event', e.g. from a philosophic [20] or business perspective [7]. An event is typically a service request by an individual or business, but it can also be initiated by a system based on for instance a timely trigger [25]. When an event occurs, individuals, organizations and systems must determine whether and how to deal with it. In urban planning, an event can be generated by a person manipulating information objects representing actual physical objects in an urban area. An Event Driven Architecture is typically combined with a Service Oriented Architecture, in which case it is called EDSOA, where a consumer of an event retrieves relevant information via a web service and submits an event itself after completing its processes [7]. An Event-Driven Architecture is typically applied in environments of loosely coupled systems [18]. As such, the combination of an event and its corresponding web service is identical to a message. We describe Event-Driven Architecture and its application in our IT infrastructure.

\subsection{Event-Driven Architecture and its Concepts}

This section introduces the relevant concepts of Event-Driven Architecture as the basis for our solution. We apply the concepts like event processing and publish/subscribe [25], [18]. Event processing distinguishes an event source and one or more event consumers (sinks). Whereas a source publishes an event, a sink can subscribe to that event. Each event may have a type and can have data as part of its body. The data is called the 'shape' of an event. Conceptually, an event type consists of a unique name, its shape and optionally metadata giving information regarding the production of an event (e.g. timestamp and source). In practice, the unique name of an event type can be composed by the existence of values of one or more fields in its shape, e.g. an event type indicates a change in the physical environment which is the addition of a building in which case a building and the relevant operation needs to be known. Such an event type may trigger a noise calculation model. In case the addition of the building leads to more inhabitants of an area, traffic calculation will be triggered. The shape of an event can be retrieved by a sink based on SOA, which leads to an EDSOA [25], [7]. In such architectures, data is retrieved from its source by a reference given in an event. A reference could be given to an alternative source than the event source. Such an alternative source might be a shared database. Messages for exchanging data are another implementation of EDA. Messages normally combine an event of a type, called 'message type', control information like sender (equal to source) and a message timestamp, and data [18]. Both events and messages need to be structured syntactically, e.g. by XML Schema. Thus, an EDA can be realised by different architectural approaches and technology. 
Events of a type are exchanged via a channel between a source and one or more sinks. A channel can have various properties like a filter for specific event types, syntax and event transformation, and a binding. An event filter is a means to enable that a sink is subscribed only to particular events. In case a sink is subscribed to all types of events, such a channel is normally known being part of a broadcasting solution [18]. Syntax transformation allows that an event and its shape are received in a format that can be processed by a sink. Event type transformation allows that an event type defined by two or more fields is reduced to an event type relevant to a recipient. A binding is typically to, for instance, a web service with its underlying communication protocol. The channel from a source to publish data can differ from the channel used by a sink, e.g. it can have a different binding and use a different syntax. An Enterprise Service Bus implements channels and the various functionality required for sources and sinks in so-called adapters [7].

Event processing is basically applied for asynchronous data sharing and hides routing information from an event source. Whenever a source publishes an event, that source can continue its processes and make data available to zero, one or more sinks. An event source is not aware which sinks have subscribed to its events and continues its processes, independent whether or not a sink consumes the data according to a mechanism like SOA. On the other hand, event sinks only receive those events to which they have subscribed. Sinks can collect data at the time it is required for their processes. Another advantage of EDA, which can also be implemented in messaging solutions, is syntax transformation. Processes share or exchange data in their own syntax. Web services are according to a specific syntax, so syntax transformation is not supported. On the other hand, EDSOA and other ways of implementing the architecture allow the application of different communication protocols.

From a business perspective, event processing does not require any design and coordination of processes over sources and sinks. Sources and sinks are said to be 'loosely coupled' [18]. EDA provides an easy mechanism to add new sources/sinks, without affecting already existing sources/sinks. Thus, the total systems can be easily extended with new functionality and a flexible IT infrastructure is provided. Of course, in case the new sources/sinks require data that is not yet available by existing sources/sinks, extensions to the latter ones have to be provided.

\subsection{Urban Strategy: Application of EDA for Urban Planning}

The above introduced concepts are the basis for the environment that supports design of an urban plan in line with the various assessments of that plan. The environment is called Urban Strategy. Starting point was to use existing state-of-the art calculation models that were already implemented by different organizational units within TNO, the Dutch organization for applied research. These models cover for instance noise and air pollution calculation. In order to be able to let different models cooperate, two main steps were taken:

1. Data: creating one data model in order to share data between systems that can function as event source and event sink;

2. Event types and an Event Service Bus: adopting EDA as the basis for communication between various models thus loosely coupling the various models.

To support real-time calculation of the impact of a change in a design and let the total infrastructure operate interactively from a user's perspective, the response time of calculation models had to be increased and the models had to be able to act as an event source and sink. Originally, the models were stand alone systems supporting analysis of specific features of an area. Response times were not really considered, since calculation was done only once for the assessment aspect supported by the model. Applying an event driven architecture implies that calculation by a model had to be performed more than once, since the output of different models may have impact on each other until a fairly stable situation is reached (see further). The generation of an event by a model based on its calculation might cause another model that subscribes to that event to produce another event. This latter event is input to the original model, leading to a new calculation. Thus, response times of models had to be increased, since more than one calculation has to be performed in a limited time frame.

Each of the models runs in its separate hardware environment. Each of these hardware environments can be easily extended according to concepts of cloud computing (in this particular case at platform level [5]). Each of these models is owned by an organizational unit and based on Internet protocols channels, had to be constructed for communication between these models operating at different physical locations and owned by different units. In this way, an environment operated by several owners is constructed, which is one of the features of a grid [5]. The user interface also acts as an event source and sink. Figure 3 visualizes the Urban Strategy architecture. This architecture is currently extended to support other types of applications like crisis management. Models and the user interfaces have data requirements. The database only exchanges data and no events. For its implementation, the data has been separated from the event types and event sources and sinks directly access their part of the database. When making a change in the database, a system component generates a related event. For example, if the traffic model calculates a new intensity on a road, this data is written in the database. Subsequently, an event is generated that is received by all event sinks that are subscribed to this event. For example, when the traffic model changes the traffic intensity on a road, the air quality model responds to the event by recalculating emissions and resulting emission levels from this road. 


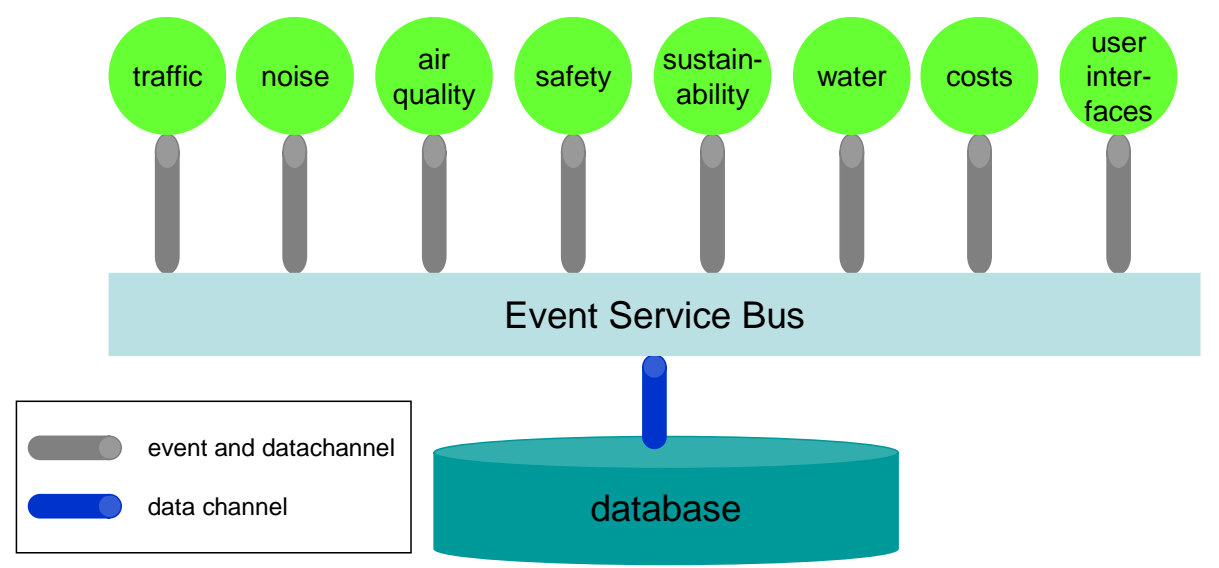

Figure 3: Software architecture of urban strategy

Event types are not completely distinguished from their data. Events are for instance directly generated by changes of data in the database. So, a database change generates a new event. Like the previous example illustrates, the air pollution model subscribes to any changes in one of the parameters that is input to its model. Thus, each model has a profile of input parameters as the basis for its subscription. After having changed data in the database, the system generates an event indicating that this data is changed. Each system component that has one or more of the parameters in its profile receives an event to indicate that the data has been changed. It implicates also that any changes in output of a model can directly be visualized on a user interface.

There is a potential danger of cycles which causes endless generation of events, e.g. an event source triggers a sink, which triggers another sink that eventually triggers the source of the initial event. The infrastructure contains various means to avoid cycling. Firstly, an initial event is always triggered by an end-user changing data representing physical reality via a 2/3 dimensional interface. An end-user generates events via the database. This end-user views the calculation results of the different models, decides that the results are sufficient and stops a cycle. Secondly, in case recalculation by a model does not give new results or changes are within certain predefined boundaries of a model, such a recalculation will not produce a new event and a cycle is broken. Thus, each model only produces changes in the database if these changes are not within predefined boundaries of accuracy.

In order to create an overall database, the specification of the input data of separate models had to be aligned and combined with user interface requirements. An overall data model is constructed to describe all the objects relevant to all event sources and sinks. The definition of each object is thus the same for each model. For example: noise calculations require detailed and accurate information of the geography of roads while the traffic model needs travel times from node to node on a coarser network. Also the output of the traffic model (overall traffic flows during rush hours) had to be transformed into the input data required by the noise model (traffic flows per day, evening, night for different vehicle classes). Therefore, the output of the traffic model can be directly used as input for the noise model.

The Urban Strategy data model supports a terrain (level of detail 0 in CityGML) and a block (level of detail 1 in CityGML) structure model required by the user interface. The user interface supports viewing and manipulating data with three interfaces: one dimensional model supporting all types of graphs, two dimensional terrain and three dimensional block structure models. The one dimensional interface visualizes different indicators as graphs. The two dimensional interface is a terrain model interface that enables a user to view, select and edit two dimensional objects like areas, roads or footprints of buildings. With this interface, an operator can specify scenarios for model calculation by adding, deleting or changing features of objects. For instance, road attributes can be changed (such as the road surface type or speed); roads can be added or closed. The building configuration can be altered and noise barriers can be added. The three dimensional interface provides a view of the city with a block structure, including landmarks for orientation (level of detail 2 in CityGML), and model output with a geographical component, such as noise contours. These noise contours can possibly also be represented as application domain extensions in CityGML. The object types stored in the database not only reflect input requirements of models, but also represent the functionality supported at the user interface. For instance, noise patterns can change by placing noise reduction screens. Thus, noise reduction screens with particular features are object types in the database (note that these are not object types in CityGML).

\section{Case Study: A1 Motorway}

This section presents one of the cases supported by the architecture implemented by Urban Strategy as described before. Firstly, it describes the objective of the case, secondly it introduces the stakeholders thereafter followed by the process, thirdly, the improvements to the current situation are presented, and finally the strong and weak points of applying Urban Strategy in this case are presented. 
The case study considers different scenario's for the A1 motorways on the stretch between Amsterdam and Gooi region. This particular motorway crossing the river Vecht needs to be widened to cater for the traffic from and to Gooi and further east and from and to the town Almere. Figure 4 visualizes the area that is subject of this case.

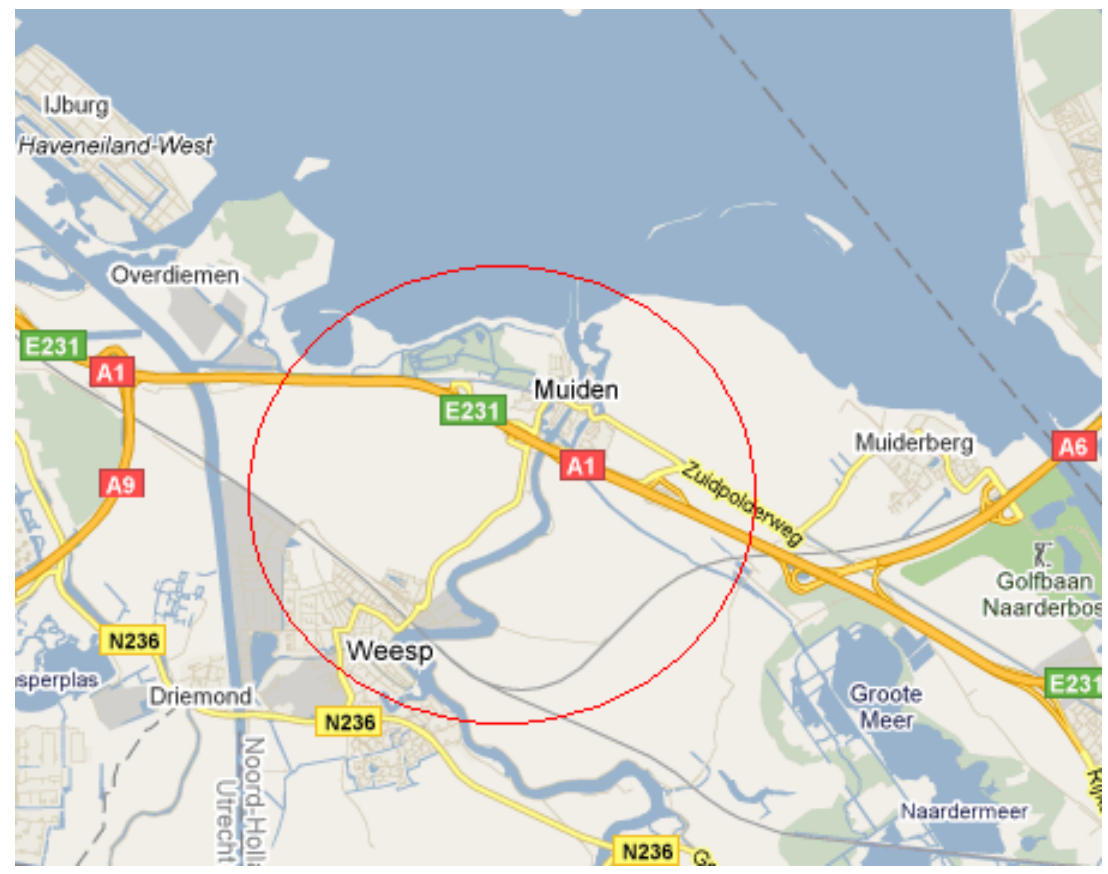

Figure 4: The A1 motorways at Muiden and Weesp

The planning of widening the A1 is commissioned by a special project office of the Dutch Department of Transport and Traffic. Furthermore, a complete new urban area is planned alongside this particular stretch between the towns Muiden and Weesp. The vision of this new area has already been produced. To realize their particular plans regarding the new urban area, the municipalities of Muiden and Weesp are the other stakeholders regarding the change of the A1 motorway. Thus, the project office and the two municipalities are the stakeholders of this case.

The project office of the Dutch Department of Traffic and Transport has been installed as the result of a recommendation of an external commission (Commission Elverding) with the objective to reduce the duration of the planning cycle in large scale infrastructure projects that effect several municipalities, e.g. new roads or potential large scale changes to existing road like the A1 motorway, new rail infrastructures, and the Rotterdam Maasvlakte. The latter project is a good example of a complex, large scale infrastructure project with the objective to (1) extend the capacity of the Port of Rotterdam in terms of container transhipment and (2) be able to handle large container vessels. For this purpose, a new terrain is constructed in the sea near the shores. The complexity of this project is not only in winning land from the sea, but also dealing with the many stakeholders involved like the users of the new terrain (stevedores, shipping lines, industry), Rijkswaterstaat responsible for road infrastructure, Port Authority, and the municipality, but also individuals and groups seeking the interests of flora and fauna in the current area.

To reduce duration of planning cycles, planning needs to be integral and faster, still taking into account the various stakeholders and ensuring participation. One of the first actions of the project office focuses on improving the calculation of traffic, air pollution, noise and sustainability of the so-called MIRT plan process (Infrastructure, Space and Transport Program spanning multiple years (Dutch MIRT: Meerjarenprogramma Infrastructuur, Ruimte en Transport)). During the vision activity (see before) several scenarios should be evaluated quite quickly. In this respect, visioning has been decomposed in two parts: the first part results in three favourable scenarios and the second should lead to a scenario that has the most support of all stakeholders involved. The project office intends to use quick scan instruments to improve efficiency and effectiveness of planning.

As the municipalities Muiden and Weesp are stakeholders in the widening of the A1 motorway in relation to their vision to create a new urban area alongside the A1 motorway, they commissioned TNO to examine the consequences of widening plan produced by the project office and investigate the impact on the local situation such as access roads, the environment, possible housing, etc. Politicians of these municipalities wanted to know the impact of the A1 motorway widening to urban environment and, if possible, try to influence the proposed changes. Changes in the motorway could possibly lead to more environmental noise and more pollution, but also changes in traffic flows could affect the sustainability and possibly the safety within the local urban area and thus affect the welfare of their citizens. All relevant data was gathered and loaded into the system, the responsible persons of both municipalities were inquired with respect to all the aspects in the planning area and their specific points of concern. 
Two interactive sessions with majors, alderman and councils of the municipalities were conducted. During these sessions, different implementation scenarios were interactively explored. Different solutions such as noise reduction screens with different heights, lowering a particular stretch of A1 motorway that crosses both municipalities and passes the river below ground level, etc. were explored. By combining different types of solutions, a favourable scenario was developed based on consequences for noise, air quality and accessibility. Both municipalities offered this scenario as a consistent proposition towards the project office of the Department of Traffic and Transport. Their solution was accepted by the project office.

The first phase of the project with the focus to come to a favourable solution for the municipalities took six weeks, which was mainly due to the availability of persons for interviews and planning and performing two interactive sessions. The total duration of the project including also the second phase of acceptance of the favourable scenario by the project office was four months. Without tools like Urban Strategy the calculation of the effects on the different aspects of one scenario would take this time, which urges most of the municipalities to only consider one scenario. The latter implies that different solutions of different scenarios can not be combined to a favourable scenario. Furthermore, the project office considers the approach in this case study as a means to reach its objectives and intends to apply Urban Strategy as one of the tools in planning of other large scale infrastructure projects.

This particular case has three strong points. First of all, the case shows that within 6 weeks from its start the municipalities got a result based on strong points from different scenarios, whilst it would normally take about 2 year to analyse, discuss, and report. As a result, secondly, the municipalities could claim 100 million Euro of the budget of the national program, which was rewarded due to the underlying proof provided by the tools. Finally, the approach taken illustrates that planning such a large scale project at national level needs to involve local participation, which can be done without a great loss of time as illustrated in this particular case.

One of the weak points is the acceptance of the IT infrastructure in large scale national projects with many (local) stakeholders as illustrated by the socio-technical system approach illustrated in the introduction of this paper. As illustrated by the case, IT infrastructure is one innovative artefact, which means that the models are an integral part of the infrastructure. As the national project office considers applying Urban Strategy for local participation in their large scale projects, local government organisations or their engineering companies would like to apply their own calculation models. Large engineering companies and municipalities, for instance, have their traffic models and would like to apply these models instead of using the ones of Urban Strategy. Not being able to replace a calculation model within the current environment by a preferred model of an end-user, lowers acceptance of the IT infrastructure and gives a discussion on perception of quality for different calculation models.

\section{Conclusions and Further Research}

Applying the new artefact called Urban Strategy based on integrating existing artefacts (models) by event driven architecture to support urban planning leads to a drastic reduction of the duration of planning processes and has contributed to improved decision making. Since data is stored in a structured way in a database, it can always be reproduced for model calculation. Decisions and proposals for favourable scenarios can always be supported whilst each scenario and its effect can always be reproduced. As the IT environment is considered for planning in other large scale infrastructure as an important tool, it is expected to support reaching the objectives of the Commission Elverding (see before).

There are still issues for further research that are mainly in two directions, namely functional and technical extensions. We will discuss both hereafter.

\subsection{Functional Extensions}

Functionally, we distinguish two types of extensions, namely support of other activities in urban planning and the aspect 'time'. First of all, other activities of urban planning could be supported like visioning without detailed design and sharing data with stakeholders for participation purposes, or other processes like crisis management, planning of location of specific buildings that have to consider security aspects (e.g. embassies), etc. are considered as extensions. These type of processes require other information sources (e.g. sensor data can be used for presenting the actual status and social media to gather user information) and possibly a different type of user interface for data representation and user interaction (e.g. several stakeholders are directly involved during a crisis). Also, integration with social media for user participation, co-creation and emergency management [26] might be required. Furthermore, the environment could be extended to support (semi-)automatic decision making for permits by integration with Building Information Modelling. This type of functional extension also refers to data sharing amongst all participating stakeholders and decision making processes.

The second functional extension is the support of 'time', in two ways. Currently, different views of the data are already supported, but each model can have a different time dimension for calculation. Alignment of time between models is for further research, but could for instance be based on rule mechanisms. Time is required for simulation, e.g. not only for crisis management but also for prediction of changes in urban environments caused by urban 
planning projects with different timescales. Aligning different urban plans in time is thus the second way of supporting time, whereas 'model time' is the first.

\subsection{Technical Extensions}

Technical extensions always have to support the functionality required by processes that have to be supported by the tool. One of the main technical challenges is to make the environment open. Openness can be achieved along the following lines:

- A user is able to apply his particular model for covering a specific feature, e.g. its specific traffic model. In this particular case, each specific model can subscribe to events. However, each model will have different data requirements that need to be covered by the business data of events (this business data is stored in the database of Urban Strategy). A clear semantics of data is required in this particular application of an event driven architecture. The semantics can be specified for instance as shared ontology that is agreed globally.

- Data acquisition is a second aspect of openness. Currently, event data is shared by a database, but this data is also stored in other sources like database with objects like buildings, streets and land parcels. Reusing this data can be implemented in different ways, e.g. subscription to events may lead to initiation of services accessing this data according to a Service Oriented Architecture or data is duplicated in a database like in Urban Strategy to meet performance requirements. Data re-use of these sources implies that they have to offer complete, up to date, and correct data. To apply a tool like Urban Strategy in other processes like described in this case, also other data needs to be accessed instead of only buildings. Data regarding use of a particular urban area like economic data, social data, and statistical data needs to be accessible. Experience shows that this data is not always complete and up to date, but also different users require different views of this data. An open linked data approach can be considered in this context [8] (see the last proposal for technical extension).

- A third aspect of openness is the access of services offered by Urban Strategy or its individual models to allow implementation of Urban Strategy as an artefact in another environment. It implies that each service of an individual model or a combination of services supported by two or more models can be instantiated externally. Currently, a combination of services of more than one model is supported by selecting those models for calculation purposes. This selection should be part of the service specification supported by Urban Strategy. This combination of features defining openness implies the support of an EDSOA, Event Driven Service Oriented Architecture. The publish and subscribe mechanism coordinates the execution of the required models, whereas these models retrieve data by services. Thus, event data is implemented by services, which is in line with the underlying model of EDA.

- Data collection is a final aspect of openness. Different approaches will be investigated in this respect. There are already open standards like CityGML, (national) standards, and defacto standards like KML for visualization or representation of physical data like buildings, roads, etc. However, data with respect to actual usage of a physical environment is not standardized. Such data comprises for instance statistical data of areas like the number of inhabitants, their age, income, etc. Part of this data is already published as open data according to principles of the semantic web [3], but not all is available yet. Semantic models are required to link data from different sources to be able to use this data in urban planning.

\section{Acknowledgments}

The authors would like to acknowledge the municipalities of Muiden and Weesp for performing the case study. The research and software development presented in this paper has been funded internally by TNO.

\section{References}

[1] M. Batty, Cities and complexity: Understanding cities with cellular automata, agent-based models, and fractals. Cambridge, MA: The MIT Press, 2005.

[2] T. M. Baynes, Complexity in Urban Development and Management - Historical Overview and Opportunities, Journal of Industrial Ecology, vol. 13, no. 2, pp. 214-227, 2009.

[3] T. Berners-Lee, J. Hendler, and O. Lassila, The Semantic Web: a new form of web content that is meaningful to computers will unleash a revolution of new possibilities, Scientific American, May 2001.

[4] H. C. Borst and H. M. E. Miedema, Comparison of noise impact indicators, calculated on the basis of noise maps of DENL, Acta Acustica united with Acustica, vol. 91, no. 2, pp. 378-385, 2005

[5] R. Buyya, C. Shin Yeo, S. Venugopal, J. Broberg, and I. Brandic, Cloud computing and emerging IT platforms: vision, hype, and reality for delivering computing as a $5^{\text {th }}$ utility, Future Generation computer systems, vol. 25, no. 6, pp. 599-616, 2009. 
[6] Calculation and measurement rules for traffic noise, Department VROM, 2002.

[7] D. Chappel, Enterprise Service Bus, O'Reilly Media, 2004.

[8] S. Dawes, Strategies for Open Government: toward a new research agenda, in Proceedings of 9th IFIP WG 8.5 International Conference on Electronic Government, Lausanne, 2010.

[9] Directive 2001/42/EC, Strategic Environmental Assessment, 2001.

[10] G. P. J. Dijkema and L. Basson, Complexity and Industrial Ecology, Foundations for a Transformation From Analysis to Action, Journal of Industrial Ecology, vol. 13, no. 2, pp. 157-164, 2009.

[11] 85/337/EC, Council Directive of 27 June 1985 on the assessment of the effects of certain public and private projects on the environment, revision M3, 2009.

[12] EU, Report from the Commission to the Council, on the application and effectiveness of the EIA Directive (Directive 85/337/EEC, as amended by Directives 97/11/EC and 2003/35/EC), 2009.

[13] G. Forestier, A. Puissant, C. Wemmert, and Gançarski, Knowledge-based region labeling for remote sensing image interpretation, to be published in Knowledge Based Systems, Elsevier, 2010.

[14] M. Fuijta, P. Krygman, and A. J. Venables, The spatial economy: Cities, regions, and international trade. Cambridge, MA: MIT Press, 1999.

[15] F. Grazi and J. C. J. M. van den Bergh, Spatial organization, transport, and climate change: Comparing instruments of spatial planning and policy, Ecological Economics, vol. 67, no. 4, pp. 630-639, 2008.

[16] A. R. Hevner, S. T. March, J. Park, and S. Ram, Design science in information systems research, MIS Quarterly, vol. 28, no. 1, pp. 75-105, 2004.

[17] G. Hoek, B. Brunekreef, S. Goldbohm, P. Fischer, and P. A. van den Brandt, Association between mortality and indicators of traffic-related air pollution in the Netherlands: a cohort study, The Lancet, vol. 360, no. 9341, pp. 1203-1209, 2002.

[18] W. J. Hofman, EDI, Web Services and ebXML, interactions in organizational networks, Uitgeverij TuteinNolthenius, 2003.

[19] G. Hophe, B. Woolf, Enterprise Integration Patterns- designing, building, and deploying messaging solutions, Addison-Wesley, 2004.

[20] P. A. L. M. Janssen, and R. A. Jekel, IMRO: Information Model for Spatial Planning, Geo-Info, 2006.

[21] K. Jaegwan, Supervenience and Mind: Selected Philosophical Essays, New York: Cambridge University Press, 1993.

[22] C. Metral, R. Billen, A-F. Cutting Decelle, and M. van Ruymbeke, Ontology-Based Models for Improving the Interoperability of 3D Urban Information, in Future of Urban Ontologies, in Proceedings of the of the Final Conference of the COST Action C21 - Towntology: Urban Ontologies for an Improved Communication in Urban Development Projects, Liège, 2009.

[23] I. Nikolic, G. P. J. Dijkema, and K. H. van Dam, Shaping the evolution of sustainable large-scale socio-technical systems, In The dynamics of regions and networks in industrial ecosystems, 2009, pp. 156-178.

[24] J. D. Ortúzar, L.G. Willumsen, Modelling Transport. USA: Wiley \& Sons, 2001.

[25] S. Overbeek, B. Klievink, and M. Janssen, A flexible, event-driven, Service-Oriented Architecture for orchestrating service delivery, IEEE Intelligent Systems, September-October 2009.

[26] H. Rossnagel, J. Zibuschka, J. Muntermann, Design of a mobile service platform for public events: improving citizens satisfaction and emergency management, in Joint proceedings of ongoing research and projects of IFIP EGOV and ePart 2010, Trauner Verlag, 2010.

[27] R. Smit , Mieghem, Hensema, Rabé, Eijk, VERSIT+ Emission parameters for calculation method 1 (CAR II), TNO rapport MON-RPT-033-DTS-2007-00709, 2007.

[28] A. Stadler, C. Nagel, G. König, T. H. Kolbe, Making interoperability persistent: A 3D geo database based on CityGML, in 3D Geo-Information Sciences (J. Lee, S. Zlatanova, Eds.). Springer, 2009, pp. 175-192.

[29] J. Unger, Z. Sümeghy, and J. Zoboki, Temperature cross-section features in an urban area, Atmospheric Research, vol. 58, no. 2, pp. 117-127, 2001.

[30] P. Wadell, UrbanSim: modelling urban development for land use, transportation and environmental planning, Journal of the American Planning Association, vol. 68, no. 3, pp. 297-314, 2002.

[31] UNECE, Draft protocol on civil liability and compensation for damage caused by the transboundary effects of industrial accidents on transboundary water, United Nations, Kiev, Ukraine, MP.WAT/2003/1, 2003. 